\title{
Association of Appendicular Skeletal Muscle Mass and Central Obesity Parameters with Lipid Profiles in Older Women
}

\author{
Lazuardhi Dwipa $^{1 *(D)}$, Syarief Hidayat ${ }^{1}$, Senov Eka Permadi ${ }^{1}$, Evan Susandi ${ }^{1}$,, Alif Bagus Rakhimullah ${ }^{2,3}$, Yuni Susanti Pratiwi ${ }^{4}$ \\ ${ }^{1}$ Department of Internal Medicine, Faculty of Medicine, Universitas Padjadjaran, Dr. Hasan Sadikin General Hospital, Bandung, \\ Indonesia; ${ }^{2}$ Department of Internal Medicine, Faculty of Medicine, Universitas Padjadjaran, Bandung, Indonesia; ${ }^{3}$ Management \\ Program, Postgraduate School, Universitas Islam Bandung, Bandung, Indonesia; ${ }^{4}$ Department of Biomedical Science, Faculty \\ of Medicine, Universitas Padjadjaran, Bandung, Indonesia
}

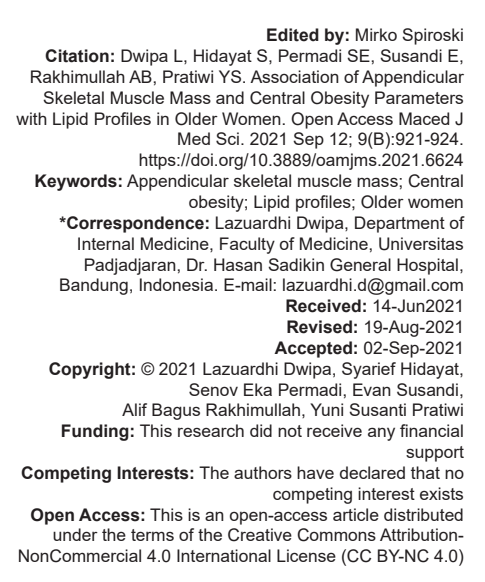

\section{Introduction}

Metabolic syndrome, characterized by central obesity, dyslipidemia, hypertension, and insulin resistance, is an important risk factor for cardiovascular disease. Various studies have reported that metabolic syndrome increases the risk of cardiovascular death by 2.5 times and 5 times the risk of developing diabetes. Elderly population is considered as a susceptible population in developing metabolic syndrome. Based on the WHO report, the prevalence of metabolic syndrome in the elderly is $11-43 \%$, the Third Report of the National Cholesterol Education Expert Panel estimates it is $40 \%$ and reaches $55 \%$ according to the National Cholesterol Education Program [1], [2], [3]. In Indonesia, a country with high population of older adults, the prevalence of metabolic syndrome among older women is $18.2 \%$ higher than men [4].

Dyslipidemia, as part of the metabolic syndrome, is a risk factor for cardiovascular disease. It is characterized by an increase in total serum cholesterol level, low-density lipoprotein (LDL) cholesterol, or triglyceride (TG) and a decrease in high-density lipoprotein (HDL) cholesterol level. This condition leads to lipid deposit formation, particularly apo-B-containing lipoprotein particles in the subintimal artery wall which triggers a complex inflammatory process, leading to atherosclerotic plaque formation [5]. Interestingly, dyslipidemia is more common in women due to hormonal changes which have a significant effect on lipoprotein metabolism, especially in older women with a higher atherogenic risk than men [6].

The process of aging induces progressive declining of muscle mass and strength which is called as sarcopenia. A decrease in resting metabolic rate and decreased physical activity occurs in sarcopenia. Both of these conditions generate fat accumulation, especially central part of the abdominal. Moreover, there is an increase of adipocytes in adipose tissues occurs in obesity. Adipocytes actively secrete leptin and various pro-inflammatory cytokines and both conditions stimulate muscle catabolism, thus a vicious cycle occurs which will accelerate sarcopenia and weight gain, mostly 
in the form of fat. The condition of decreased muscle mass and increased visceral fat in the older population is called as sarcopenia obesity which increases the risk of developing metabolic syndrome and cardiovascular disease in older adults, due to its association with proinflammatory conditions [7].

There are limited studies assessing the effect of body composition as a cardiovascular risk factor in the older women, particularly in the form of appendicular skeletal muscle mass (ASMM) which describes sarcopenia and central obesity on metabolic disorders, particularly the lipid profile as a cardiovascular risk factor. The previous studies are limited using grip strength as a predictor to determine muscle mass and strength and abdominal circumference and body mass index (BMI) as markers of obesity are still contradictory, especially for each lipid profile. Several studies suggest that decreased muscle strength and high level of fat mass are associated with an increase in total cholesterol, TG, and LDL level and a decrease in HDL level, but other studies showed different results [8], [9]. This study is aimed to determine the relationship between ASMM and central obesity with the respective lipid profiles in older women.

\section{Materials and Methods}

This was a correlative analysis study with cross-sectional method. We conducted the study to the outpatients at geriatric clinic of Hasan Sadikin General Hospital, Bandung, from January 2019 to February 2020. This study has approved by the Medical Research Ethics Committee of Hasan Sadikin General Hospital, Bandung LB.02.01/X.6.5/48/2020).

Data were obtained from medical records. Inclusion criteria for subjects are consisted of subjects aged $>60$ years with complete demographic data such as age, gender, anthropometric data (height, weight, abdominal circumference, calf circumference, and $\mathrm{BMI}$ ), body composition measurement results in the form of ASMM, and truncal fat mass (TrFM) based on bioelectrical impedance analysis (BIA) examination and laboratory results of lipid profile taken at the same time.

Geriatric Clinic of Hasan Sadikin General Hospital has a standard procedure of body composition examination. Body composition was measured using Tanita MC-980 (Tanita Corp., Tokyo, Japan). It provides a complete measurement of weight, BMI, body fat, and fat mass percentage, and fat-free mass, including segmental analysis of each body part for muscle mass and fat mass necessary for this study. For each $B I A$, sex, age, and height were directly inputted into the instrument before the impedance measurement. Testing was scheduled to allow for a 10-12 $\mathrm{h}$ fasting window, avoid to strenuous exercise for $4 \mathrm{~h}$ or more, avoid to drink for $1 \mathrm{~h}$, and patients were asked to void their bladder before testing to optimize the accuracy. Any metal items were removed from the patients. Then, subject's feet were guided onto the BIA foot sensors by the examiner to ensure optimal contact and centralized the placement of heel. All BIA measurements were completed by a trained examiner according to the device manufacturers' instructions. All assessors in our clinic have been standardized.

The baseline characteristics of subjects were presented in numbers and percentage for categorical data, while for numerical data, normality test was performed using Kolmogorov-Smirnov. Data were presented in mean with standard deviation or median with ranges (min-max). Log transformation was performed to each non-normally distributed data before the bivariate analysis. Bivariate analysis was performed after univariate analysis was done. Pearson correlation was used to analyze the relationship of the variables. Each Pearson's correlation test with $p<0.05$ was considered to be significant. Statistical analysis was done using SPSS 25.0 program.

\section{Results}

A total of 61 subjects were included in the inclusion criteria in this study. The subject characteristics are described in Table 1 which shows that the mean age of the subjects was $69 \pm 5$ years. The mean of BMI was $25.8 \pm 4.5$ with a normal BMI percentage of $44.2 \%$ and obesity of $16.4 \%$. The mean of abdominal and calf circumference was $89 \pm 10 \mathrm{~cm}$ and $35 \pm 4 \mathrm{~cm}$, respectively. The mean of ASMM was $8.27 \pm 1.29 \mathrm{~kg} / \mathrm{m}^{2}$ and TrFM was $10.98 \pm 3.92 \mathrm{~kg} / \mathrm{m}^{2}$.

Table 1: Subject characteristics

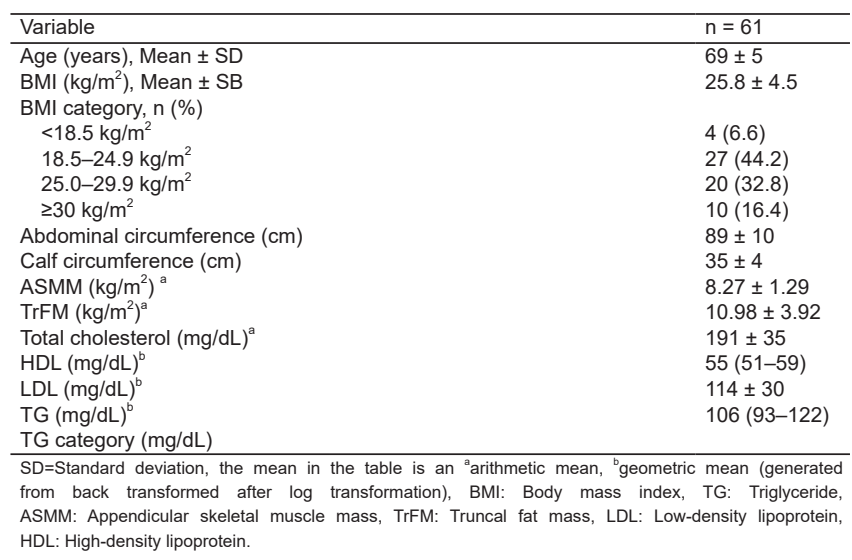

Table 2 indicates that there was a significant relationship between appendicular muscle mass and HDL with weak negative correlation $(r=-0.297, p=0.01)$ and a significant relationship between TrFM and TG with a moderate correlation $(r=0.339, p=0.004)$. There was no significant relationship between calf 
circumference and abdominal circumference to lipid profile parameters.

Table 2: ASMM and central obesity to lipid profiles

\begin{tabular}{lllll}
\hline TrFM & Total cholesterol & Log HDL & Log LDL & Log triglyceride \\
\hline Calf circumference & & & & \\
$\quad r$ & -0.129 & -0.031 & -0.094 & -0.032 \\
p & 0.161 & 0.404 & 0.236 & 0.402 \\
Abdominal circumference & & & & \\
r & 0.117 & -0.058 & 0.102 & 0.211 \\
p & 0.184 & 0.330 & 0.218 & 0.052 \\
ASMM & & & & \\
r & 0.035 & $-0.297^{*}$ & 0.183 & -0.022 \\
p & 0.395 & 0.01 & 0.079 & 0.432 \\
TrFM & & & & \\
r & 0.084 & -0.155 & 0.145 & $0.339^{*}$ \\
p & 0.259 & 0.117 & 0.132 & 0.004 \\
\hline Analysis using Pearson's correlation, *significant if $p<0.05$, ASMM: Appendicular skeletal muscle mass,
\end{tabular}

TrFM: Truncal fat mass, LDL- Low-density lipoprotein, HDL: High-density lipoprotein.

Table 3 shows a significant relationship between abdominal circumference and TrFM with moderate positive correlation $(r=0.579, p<0.001)$ and a significant relationship between calf circumference and ASMM with weak positive correlation $(p=0.041$, correlation coefficient $=0.225$ ) .

Table 3: Correlation of calf circumference to appendicular muscle mass and abdominal circumference to TrFM

\begin{tabular}{lll}
\hline Calf circumference & ASMM & \\
\hline & $\mathrm{R}$ & $\mathrm{p}$-value \\
& 0.225 & $0.041{ }^{*}$ \\
\hline Abdominal circumference & $\mathrm{TrFM}$ & $\mathrm{p}$-value \\
\hline & $\mathrm{R}$ & $<0.001{ }^{*}$ \\
\hline${ }^{*}$ Pearson correlation, significant if $\mathrm{p}<0.05$, ASMM: Appendicular skeletal muscle mass, TrFM: Truncal fat
\end{tabular}

mass.

\section{Discussion}

Appendicular muscle mass and TrFM did not have a significant relationship to total cholesterol and LDL in the results of this study. Total cholesterol and LDL are not always related to body composition, although obesity increases the risk of total and LDL cholesterol levels to rise, there are several other things that can affect the levels of these two parameters including age, nutritional intake, and level of physical activity which, in this study, were not assessed, hence, it becomes the limitations of the study [10], [11].

In this study, the result of appendicular muscle mass was negatively related to HDL level, the higher the $\mathrm{HDL}$, the lower the appendicular muscle mass. It might be contradictory at first but actually it could be explained by looking at its muscle fibers type and its proportion. Sarcopenia is characterized by a progressive decrease in muscle mass characterized by changes in muscle fiber composition, especially a decrease in type 2 muscle fibers and also its conversion to type 1 muscle fibers. Type 2 muscle fibers are fast-twitch type that was used on high intensity but short duration activities which involve glycolytic process as a way in utilizing energy. On the other hand, type 1 or slow-twitch type muscle fibers have lipid oxidation properties that for long duration type activity utilizing fatty acid (lipid) as its source of energy which could be reflected based on the level of HDL [12], [13]. Hence, higher level of $\mathrm{HDL}$ in a person might also reflect a good quality and quantity of type 1 muscle fiber. Most older women in our study are in fact in good level of daily activity reflected by its normal level of HDL which shown in base line characteristics in Table 1. Therefore, there might be a certain compensatory mechanism in the muscle fibers in older women to maintain its function in daily living activity despite its decrease in total mass due to aging process. Type I muscle fibers might increase in proportion or remain unchanged while type II muscle fibers decrease [12]. However, this would need to be proven further by performing muscle biopsy to know the exact proportion of each muscle fiber.

In this study, there was a positive relationship between TrFM and TG, this result is in line with the study by Lim et al. with a multiethnic study in Japan, it shows that Asian women have higher abdominal and visceral adipocytes than Caucasian women with the same BMI [14]. Hypertriglyceridemia is the most common dyslipidemia condition in obesity caused by an increase in adipocytes and the release of free fatty acids results in the accumulation of TG [15].

Calf circumference and abdominal circumference as body anthropometric parameters, both are a very simple and easy to measure to be applied in any clinical setting. Thus, the usefulness of these parameters as screening tools to reflect the conditions of lipid profile has come to attention. Based on the results of the analysis in this study, calf circumference and abdominal circumference did not have a significant relationship with lipid profile. However, there was a significant correlation between calf circumference to ASMM and abdominal circumference to TrFM which implies that calf circumference and abdominal circumference were indirectly related to lipid profile. This shows that calf circumference and abdominal circumference might be still be useful to reflect muscle mass and visceral fat as body composition parameters but could not directly reflect the level of lipid profile abnormalities in the clinical setting.

As a limitation, this is a cross-sectional study which used secondary data from medical records, hence, factors which could affect the results of the study such as changes in body weight, types of physical activity, consumption of cholesterol-lowering drugs, and nutritional intake could not be assessed.

\section{Conclusion}

Appendicular muscle mass has a negative relationship with HDL level and TrFM has a positive relationship with TG level in older women as a precaution against cardiovascular risk. 


\section{References}

1. van Ancum JM, Jonkman NH, van Schoor NM, Tressel E, Meskers CG, Pijnappels $\mathrm{M}$, et al. Predictors of metabolic syndrome in community-dwelling older adults. PLoS One. 2018;13(10):e0206424. https://doi.org/10.1371/journal. pone.0206424

PMid:30379967

2. Bayturan O, Tuzcu EM, Lavoie A, Hu T, Wolski K, Schoenhagen $\mathrm{P}$, et al. The metabolic syndrome, its component risk factors, and progression of coronary atherosclerosis. Arch Intern Med. 2010;170(5):478-84. https://doi.org/10.1001/ archinternmed.2009.551

PMid:20212186

3. Arai $\mathrm{H}$, Yamamoto $\mathrm{A}$, Matsuzawa $\mathrm{Y}$, Saito $\mathrm{Y}$, Yamada $\mathrm{N}$, Oikawa S, et al. Prevalence of the metabolic syndrome in elderly and middle-aged Japanese. J Clin Gerontol Geriatr. 2010;1:42-7.

4. Kamso S. Prevalence of metabolic syndrome in older Indonesians. Asia Pac J Public Health. 2008;20:244-50.

PMid:19533888

5. Hedayatnia M, Asadi Z, Zare-Feyzabadi R, YaghootiKhorasani M, Ghazizadeh $\mathrm{H}$, Ghaffarian-Zirak $\mathrm{R}$, et al. Dyslipidemia and cardiovascular disease risk among the MASHAD study population. Lipids Health Dis. 2020;19(1):42. https://doi.org/10.1186/s12944-020-01204-y

PMid:32178672

6. Phan BAP, Toth PP. Dyslipidemia in women: Etiology and management. Int J Womens Health. 2014;6:185-94.

PMid:24532973

7. Kim TN, Choi KM. The implications of sarcopenia and sarcopenic obesity on cardiometabolic disease. J Cell Biochem. 2015;116(7):1171-8. https://doi.org/10.1002/jcb.25077 PMid:25545054

8. Baek SJ, Nam GE, Han KD, Choi SW, Jung SW, Bok AR, et al. Sarcopenia and sarcopenic obesity and their association with dyslipidemia in Korean elderly men: The 2008-2010 Korea national health and nutrition examination survey.
J Endocrinol Invest. 2014;37(3):247-60. https://doi.org/10.1007/ s40618-013-0011-3

PMid:24615361

9. Vella CA, Nelson MC, Unkart JT, Miljkovic I, Allison MA. Skeletal muscle area and density are associated with lipid and lipoprotein cholesterol levels: The multi-ethnic study of atherosclerosis. J Clin Lipidol. 2020;14(1):143-53. https://doi.org/10.1016/j. jacl.2020.01.002

PMid:32061531

10. Ferrara A, Barrett-Connor E, Shan J. Total, LDL, and HDL cholesterol decrease with age in older men and women. The rancho bernardo study 1984-1994. Circulation. 1997;96(1):3743. https://doi.org/10.1161/01.cir.96.1.37 PMid:9236414

11. Gostynski M, Gutzwiller F, Kuulasmaa K, Döring A, Ferrario M Grafnetter D, et al. Analysis of the relationship between total cholesterol, age, body mass index among males and females in the WHO MONICA project. Int J Obes Relat Metab Disord. 2004;28(8):1082-90. https://doi.org/10.1038/sj.ijo.0802714 PMid:15211364

12. Tikkanen $\mathrm{HO}$, Näveri $\mathrm{H}$, Härkönen $M$. Skeletal muscle fiber distribution influences serum high-density lipoprotein cholesterol level. Atherosclerosis. 1996;120(1-2):1-5. https:// doi.org/10.1016/0021-9150(95)05652-1

PMid:8645350

13. Miljkovic N, Lim JY, Miljkovic I, Frontera WR. Aging of skeletal muscle fibers. Ann Rehabil Med. 2015;39(2):155-62. https://doi. org/10.5535/arm.2015.39.2.155

PMid:25932410

14. Lim U, Ernst T, Buchthal SD, Latch M, Albright CL, Wilkens LR, et al. Asian women have greater abdominal and visceral adiposity than Caucasian women with similar body mass index. Nutr Diabetes. 2011;1(5):e6. https://doi.org/10.1038/ nutd.2011.2

PMid:23449381

15. Klop B, Elte JW, Cabezas MC. Dyslipidemia in obesity: Mechanisms and potential targets. Nutrients. 2013;5(4):121840. https://doi.org/10.3390/nu5041218

PMid:23584084 